\title{
Effect of Foliar Application of Compost Tea on Soil Quality, Nutrient Uptake and Apparent Nutrient Balance in Soil under Soybean [Glycine $\max (\mathrm{L})]$
}

\author{
N. Shrinivas, P. H. Vaidya and P. H. Gourkhede* \\ Department of Soil Science and Agriculture Chemistry, Vasantrao Naik Marathwada Krishi \\ Vidyapeeth, Parbhani-431402, Maharashtra, India \\ *Corresponding author
}

\section{A B S T R A C T}

\section{Keywords}

Compost tea, foliar application,

Soybean, Yield,

Nutrient uptake and apparent nutrient balance

Article Info

\section{Accepted:}

22 April 2021

Available Online:

10 May 2021
The field experiment was conducted on Vertic Heplustepts (Inseptisols) soil of farm, Department of Soil Science and Agriculture Chemistry, College of Agriculture, Latur. The experimental plot was laid out in a randomize block design (RBD) with three replication and eight treatments viz., $\mathrm{T}_{1}$ - Control (RDF 100\%), $\mathrm{T}_{2}-100 \% \mathrm{RDF}+$ Water spray, $\mathrm{T}_{3}-\mathrm{RDF}+$ Compost tea @ 10\% foliar spray, T4 - RDF + Compost tea @ 15\% foliar spray, $\mathrm{T}_{5}-\mathrm{RDF}+$ Compost tea @ $20 \%$ foliar spray, $\mathrm{T}_{6}-\mathrm{RDF}+$ Compost tea @ $25 \%$ foliar spray, $\mathrm{T}_{7}-\mathrm{RDF}+$ Compost tea @ $50 \%$ foliar spray, $\mathrm{T}_{8}-\mathrm{RDF}+$ Compost tea @ $75 \%$ foliar spray. The results indicated that the plant nutrient content, uptake and yield of soybean were significantly increased with foliar application of compost tea. The maximum plant nutrient content, uptake and yield of soybean were recorded in treatment $\mathrm{T}_{7}-\mathrm{RDF}+$ Compost tea @ $50 \%$ foliar spray fallowed by $\mathrm{T}_{8}-\mathrm{RDF}+$ compost tea @ $75 \%$ foliar spray which was significantly superior over control (RDF). The available N P K and $\mathrm{Zn}$ in soil were found significantly decreased over control. Increasing the dose of foliar application of compost tea on soybean a decrease in available NPK status of soil after harvest of the soybean crop. From the above result however concluded that the foliar application of compost tea along with RDF increased nutrient content, uptake and yield of soybean crop and mining of nutrients from soil.

\section{Introduction}

Major soybean growing states in India are Madhya Pradesh, Maharashtra, Rajasthan, Karnataka, Uttar Pradesh, Andhra Pradesh, Nagaland, and Gujarat. Compost teas are a sustainable, economic, and feasible way to efficiently utilize nutrients from pre and post consumer food waste and vegetative wastes from modern agriculture. Compost tea is an umbrella term referring to a nutrient and microorganism rich solution prepared by releasing compost nutrients and microbiology into solution. Recently, compost teas have been recognized for their ability to suppress several foliar diseases as well as seed and root 
rot (Scheuerell 2004). A compost tea can be tailored to its desired use. The tea improves the nutrient retention of the soil thus stimulating plant growth. If your soil can retain its nutrients it helps minimize the need to use fertilizer. Increasing the nutrients available to the root system leads to a stronger healthier plant. Compost tea assists in reducing the negative impact that chemicalbased pesticides, herbicides and fertilizers have on beneficial micro-organisms in the ecosystem. Compost tea is usually used as a foliar spray or a soil drenches (Ingham, 2000). The nutrients in compost tea are available for plant uptake. Uptake of these nutrients can result in improved plant health which may reduce the need for supplemental chemicals products to sustain plant growth (Ingham, 2005). The suppression of disease through compost tea application has been extensive documented and summarized by Scheuerell and Mahaffee (2002), and Litterick et al., (2004). The compost tea quality and its effects on plant may be predicted based on compost quality (Pant et al., 2012). The use compost teas enhanced the plant yield and quality, and reduced the input costs.

\section{Materials and Methods}

This study was carried out at the research farm, Department of Soil Science and Agriculture Chemistry, College of Agriculture, Latur. The soil of the experimental site was deep, black in color with good drainage and as per USDA soil Taxonomy classification this soil belongs to Vertic Haplustepts. The required quantity of compost tea was be prepared as per standard procedure (Das, 1999) and its characterization Viz. pH, Electrical conductivity, Organic carbon, available nutrients N P K and DTPA extractable micronutrients. The experimental plot was laid out in a randomized block design (RBD). Plot size was $4 \times 5 \mathrm{~m}^{2}$ and row to row distance was $45 \mathrm{~cm}$ and plant to plant distance
$5 \mathrm{~cm}$. The popular variety MAUS-71 of soybean was used for this experiment with eight treatments viz, $\mathrm{T}_{1}$ - control (RDF 100\%), $\mathrm{T}_{2}-100 \% \mathrm{RDF}+$ water spray, $\mathrm{T}_{3}-\mathrm{RDF}+$ compost tea@10\% foliar spray, T4 - RDF + compost tea @15\% foliar spray, T5-RDF + compost tea@20\% foliar spray, $\mathrm{T}_{6}-\mathrm{RDF}+$ compost tea@25\% foliar spray, $\mathrm{T}_{7}-\mathrm{RDF}+$ compost tea@50\% foliar spray, $\mathrm{T}_{8}-\mathrm{RDF}+$ compost tea@75\% foliar spray. The foliar application of compost tea at 30, 45, 60 DAS. Content and uptake of nutrient was studied at different growth stages of soybean crop Viz. Flowering, pod formation and maturity stage and nutrients balance in soil after harvest of soybean crop were work out.

\section{Results and Discussion}

\section{Concentration and uptake of nitrogen}

The data presented in table- 1 indicated that total nitrogen content of plant samples varied significantly at flowering, pod formation and harvesting stage of crop. The concentration of nutrients decreased as the growth progressed towards harvesting of the crop. The $\mathrm{N}$ concentration in soybean plants at flowering stage, pod formation stage and harvesting stage ranged from 2.91 to $3.25 \%, 1.69$ to 1.89 $\%$ and 1.25 to $1.40 \%$ respectively. The maximum $\mathrm{N}$ concentration in flowering and pod formation stage was recorded with the treatment $\mathrm{T}_{7}-\mathrm{RDF}+$ compost tea @ $50 \%$ foliar spray at 30, 45 and 60 DAS (3.25\%, $1.89 \%$, and $1.4 \%$ respectively and in grain $5.03 \%$ ) which was at par with $\mathrm{T}_{8}-\mathrm{RDF}+$ compost tea @ $75 \%$ foliar spray at 30,45 and 60 DAS, $\mathrm{T}_{6}, \mathrm{~T}_{5}$ and $\mathrm{T}_{4}$. Whereas the treatment $\mathrm{T}_{1}$ (RDF) was found minimum concentration of $\mathrm{N}$ in soybean at flowering, pod formation stage and harvesting $(2.91 \%, 1.69 \%$ and $1.25 \%$ respectively).

The uptake of nitrogen in plant at flowering, pod formation stage and harvesting of soybean 
crop was varied from 54.67 to $78.05 \mathrm{~kg} \mathrm{ha}^{-1}$, 6.25 to $86.06 \mathrm{~kg} \mathrm{ha}^{-1}$ and 39.02 to $55.27 \mathrm{~kg} \mathrm{ha}^{-}$ ${ }^{1}$ in straw respectively and in grain 71.12 to $103.09 \mathrm{~kg} \mathrm{ha}^{-1}$. The total uptake varied from 110.14 to $158.35 \mathrm{~kg} \mathrm{ha}^{-1}$. The statistically which was at par with treatment $\mathrm{T}_{6}-\mathrm{RDF}+$ compost tea@25\% foliar spray at 30,45 and 60 DAS and $\mathrm{T}_{8}-\mathrm{RDF}+$ compost tea @ $75 \%$ foliar spray at 30, 45 and 60 DAS and significantly superior over rest of the treatments. Similar finding also reported by Hegazi and Algharib (2014) application of $25 \% \mathrm{NPK}+$ foliar application of compost tea 75 per cent increases the nitrogen content cowpea seeds. Hiradeve et al., (2011), maximum leaf nitrogen content in groundnut was observed in $400 \mathrm{ppm}$ vermicompost wash as foliar application.

\section{Concentration and uptake of phosphorous}

The data presented in Table 2 indicated that total phosphorus content of plant samples varied significantly at flowering, pod formation and harvesting stage of crop. The concentration of nutrients decreased as the growth progressed towards harvesting of the crop. The $\mathrm{P}$ concentration in soybean plants at flowering stage, pod formation stage and harvesting stage ranged from 0.24 to 0.33 per cent, 0.18 to 0.26 per cent and 0.10 to 0.17 per cent respectively. The maximum $\mathrm{P}$ concentration in flowering, pod formation and harvesting stage was recorded with the treatment $\mathrm{T}_{7}-\mathrm{RDF}+$ compost tea @ $50 \%$ foliar spray $\left(\begin{array}{llll}0.33 \% & 0.26 \% & \text { and } & 0.17 \%\end{array}\right.$ respectively and in grain 0.77 ) which was at par with $\mathrm{T}_{8}$ - RDF + compost tea @ 75 per cent foliar spray at 30, 45 and 60 DAS and treatment $\mathrm{T}_{6}-\mathrm{RDF}+$ compost tea @ $25 \%$ foliar spray at 30, 45 and 60 DAS at flowering stage. Whereas the treatment $\mathrm{T}_{1}$ (control) was found minimum concentration at flowering, pod formation and harvesting stage $(0.24 \%$, $0.18 \%$, and $0.09 \%$ respectively and in grain $0.59 \%$ ) and significantly superior over rest of the treatments. The uptake of phosphorus in plant at flowering and pod formation stage was significantly higher with the treatment $\mathrm{T}_{7}$ - RDF + compost tea @ $50 \%$ foliar spray at 30,45 and 60 DAS $\left(7.88 \mathrm{~kg} \mathrm{ha}^{-1}\right.$ and $8.69 \mathrm{~kg}$ $\mathrm{ha}^{-1}$ respectively) which was at par with $\mathrm{T}_{8^{-}}$ RDF + compost tea @ $75 \%$ foliar spray at 30, 45 and 60 DAS. The $\mathrm{T}_{1}-$ control was found with minimum uptake of $\mathrm{P}\left(4.53 \mathrm{~kg} \mathrm{ha}^{-1}\right.$ and $4.99 \mathrm{~kg} \mathrm{ha}^{-1}$ respectively).The uptake of phosphorus at harvest stage in straw, grain and total uptake was significantly higher with the treatment $\mathrm{T}_{7}-\mathrm{RDF}+$ compost tea @ $50 \%$ foliar spray at 30, 45 and 60 DAS (6.72, 16.51 and $23.23 \mathrm{~kg} \mathrm{ha}^{-1}$ respectively) which was at par with $\mathrm{T}_{8}-\mathrm{RDF}+$ compost tea @ $75 \%$ foliar spray at 30, 45 and $60 \mathrm{DAS}$. The $\mathrm{T}_{1}-$ control was found with minimum uptake of $\mathrm{P}$ $\left(2.86,9.41\right.$ and $12.26 \mathrm{~kg} \mathrm{ha}^{-1}$ respectively) and all other treatment were significantly superior to it.

Similar finding was also reported by Hegazi and Algharib (2014) they reported that 25\% $\mathrm{NPK}+$ foliar application of compost tea $75 \%$ increases the phosphorus uptake and content in cowpea seeds. Maheswari et al., (2003) foliar application vermicompost wash at 1:5 dilutions with complete dose of RDF resulted in higher $\mathrm{P}$ uptake in onion. In addition to proper mineral fertilization, bio stimulants can enhance the effectiveness of fertilizers as well as nutrient utilization from soil (Frankenberger and Arshad, 1995).

\section{Concentration and uptake of potassium}

The data presented in Table 3 indicated that total potassium content of plant samples varied significantly at flowering, pod formation and harvesting stage of crop. The concentration of nutrients decreased as the growth progressed towards harvesting of the crop. The K concentration in soybean plants at flowering stage and pod formation stage ranged from 1.29 to 1.57 per cent and 1.01 to 
1.22 per cent respectively. The maximum $\mathrm{K}$ concentration in flowering and pod formation stage was recorded with the treatment $\mathrm{T}_{7}$ RDF + compost tea @ 50\% foliar spray ( $1.57 \%$ and $1.22 \%$ respectively) which was at par with $\mathrm{T}_{8}-\mathrm{RDF}+$ compost tea @ $75 \%$ foliar spray at 30, 45 and $60 \mathrm{DAS}$, and treatment $T_{6}$ and $T_{5}$. Whereas the treatment $T_{1}$ (control) was found minimum concentration of $\mathrm{K}$ in soybean at flowering and pod formation stage $(1.29 \%$ and $1.01 \%$ respectively). The $\mathrm{K}$ concentration in soybean at harvest straw ranged from 0.75 to 0.91 per cent and in grain 1.41 to 1.71 per cent. The maximum $\mathrm{K}$ concentration in straw and grain was recorded with the treatment $\mathrm{T}_{7}-\mathrm{RDF}+$ Compost tea@50\% foliar spray at 30,45 and 60 DAS (0.91 and $1.71 \%$ respectively) which was at par with $\mathrm{T}_{8}-\mathrm{RDF}+$ Compost tea @ 75 $\%$ foliar spray at 30,45 and $60 \mathrm{DAS}^{\mathrm{T}} \mathrm{T}_{6}$ and $\mathrm{T}_{5}$. Whereas the treatment $\mathrm{T}_{1}$ (control) was found minimum concentration of $\mathrm{K}$ in soybean straw and grain $(0.75 \%$ and $1.41 \%$ respectively).

The uptake of potassium in plant at flowering and pod formation stage was significantly higher with the treatment $\mathrm{T}_{7}-\mathrm{RDF}+$ compost tea @ $50 \%$ foliar spray at 30,45 and 60 DAS (37.71 and $55.89 \mathrm{~kg} \mathrm{ha}^{-1}$ respectively) which was at par with $\mathrm{T}_{8}$ and $\mathrm{T}_{6}$. The $\mathrm{T}_{1}-$ control was found with minimum uptake of $\mathrm{K}$ (24.22 and $35.90 \mathrm{~kg} \mathrm{ha}^{-1}$ respectively) and all other treatments were significantly superior to it. The uptake of potassium at harvest stage in straw, grain and total uptake was significantly higher with the treatment $\mathrm{T}_{7}-\mathrm{RDF}+$ compost tea@ $90 \%$ foliar spray at 30,45 and 60 DAS (35.99, 35.14 and $71.13 \mathrm{~kg} \mathrm{ha}^{-1}$ respectively) which was at par with $\mathrm{T}_{8}$ and $\mathrm{T}_{6}$. The $\mathrm{T}_{1}-$ control was found with minimum uptake of $\mathrm{K}$ (23.32, 22.19 and $45.50 \mathrm{~kg} \mathrm{ha}^{-1}$ respectively) and all other treatments were significantly superior to it.

The above finding supports the observation of
Hegazi and Algharib (2014) they reported that $25 \%$ NPK + foliar application of compost tea $75 \%$ increases the potassium content cowpea seeds. Zodape et al., (2008) promotive effect of SLF as foliar application might be because of increase root proliferation and establishment, there by plants were able to mine more nutrients from distant place and deeper soil horizon, in balanced proportion.

\section{Straw and Grain yield $\mathrm{kg} \mathrm{ha}^{-1}$}

The data regarding straw yield and grain yield recorded at harvest stage was presented in Table 2. It was observed from the results that the treatment $\mathrm{T}_{7}-\mathrm{RDF}+$ Compost tea @ $50 \%$ foliar spray recorded significantly higher straw yield (3953.42 $\mathrm{kg} \mathrm{ha}^{-1}$ ) and grain yield (2050.00 kg ha ${ }^{-1}$ ) over the rest of treatments and it was at par with the treatment $\mathrm{T}_{8}-\mathrm{RDF}$ +Compost tea@75\% foliar spray and $\mathrm{T}_{6}$ RDF + Compost tea @ $25 \%$ foliar spray. The lowest grain yield was recorded by treatment $\mathrm{T}_{1}$ - control straw yield $\left(3120.30 \mathrm{~kg} \mathrm{ha}^{-1}\right)$ and grain yield $\left(1580.00 \mathrm{~kg} \mathrm{ha}^{-1}\right)$. And all other treatment were significantly superior to it. The increased straw and seed yield in soybean might be due to effect of foliar application of compost tea. Rathore et al., (2009). Foliar application of seaweed extract on soybean @ $10 \%$ which significantly higher the straw and grain yield $\left(56.97\right.$ and $17.18 \mathrm{~kg} \mathrm{ha}{ }^{-1}$ respectively) compared with other foliar treatment hegazi et al., (2014).

The results showed in cow pea field $25 \%$ $\mathrm{NPK}+75 \%$ compost tea as foliar spray significantly increase the seed weight plaot $^{-1}$ (4.70 g) compared with other foliar treatment. Mostafa et al.,(2012) reported that the yield and fruit quality significantly increased when applied compost tea which as foliar application was used on Washington navel orange trees and on Thompson seedless grapevines. 
Thind et al.,(2001) stated that availability of photo assimilates during longer grain filling period of maize combined with more leaf area at flowering stage might have accounted for yield increase under biogas slurry + panchagavya.

Apparent balance of nitrogen status of soil $\left(\mathrm{kg} \mathrm{ha}^{-1}\right)$

Data on available nitrogen $(\mathrm{N})$ status after harvest of soybean as influenced by foliar application of compost tea when studied revealed that the irrespective of treatment combinations a definite gain or loss in available nitrogen status of soil after harvest of soybean crop was observed. Maximum gain in available nitrogen status $\left(39.52 \mathrm{~kg} \mathrm{ha}^{-1}\right)$ was registered in the treatment $\mathrm{T}_{1}$-control receiving (100 \% RDF through chemical fertilizers while more loss of available N ($6.78 \mathrm{~kg} \mathrm{ha}^{-1}$ ) was observed in the treatment $\left(\mathrm{T}_{7}\right)$ where $\mathrm{RDF}+\mathrm{T}_{7}-\mathrm{RDF}+$ Compost tea @ $50 \%$ foliar spray. In general increasing the dose of foliar applied compost tea on soybean a decrease in available nitrogen status of soil after harvest of the soybean crop growth stages of compost tea this caused significant increase in the yield as well as other biometric parameters.

Table.1 Effect of foliar application of compost tea on straw yield, grain yield and total dry matter yield of soybean $\left(\mathrm{kg} \mathrm{ha}^{-1}\right)$

\begin{tabular}{|c|c|c|c|}
\hline \multirow[t]{2}{*}{ Treatment } & \multicolumn{2}{|c|}{ At harvesting stage } & \multirow{2}{*}{$\begin{array}{c}\text { Total dry } \\
\text { matter } \\
\text { Kg ha }^{-1}\end{array}$} \\
\hline & $\begin{array}{l}\text { Straw yield } \\
\mathrm{kg}^{-1} \mathrm{ha}^{-}\end{array}$ & $\begin{array}{l}\text { Grain yield } \\
\mathrm{kg}^{-1} \mathrm{ha}^{-}\end{array}$ & \\
\hline$T_{1}-$ Control (RDF 100\%) & 3120.3 & 1580 & 4700.3 \\
\hline $\mathrm{T}_{2}-100 \% \mathrm{RDF}+$ Water spray & 3167.1 & 1578.97 & 4746.08 \\
\hline$\underset{\text { T }}{\mathrm{T}_{3} \text { - RDF + Compost tea @ } 10 \% \text { foliar }}$ & 3251.35 & 1651 & 4902.35 \\
\hline T4 - RDF + Compost tea @ 15\% foliar & 3389.58 & 1730 & 5119.58 \\
\hline $\begin{array}{c}\mathrm{T}_{5}-\mathrm{RDF}+\text { Compost tea } @ 20 \% \text { foliar } \\
\text { spray }\end{array}$ & 3582.1 & 1825.99 & 5408.1 \\
\hline$\underset{\text { Tspray }}{\mathrm{T}_{6}-\mathrm{RDF}+\text { Compost tea @ } 25 \% \text { foliar }}$ & 3763.08 & 1920.03 & 5683.12 \\
\hline $\begin{array}{c}\mathrm{T}_{7}-\mathrm{RDF}+\text { Compost tea @ } \mathbf{5 0} \% \text { foliar } \\
\text { spray }\end{array}$ & 3953.42 & 2050 & 6003.42 \\
\hline $\begin{array}{c}T_{8}-\text { RDF + Compost tea @ } 75 \% \text { foliar } \\
\text { spray }\end{array}$ & 3831.73 & 2016 & 5847.73 \\
\hline General mean & 3507.33 & 1794 & 5301.3 \\
\hline $\mathrm{SE}(\mathbf{m}) \pm$ & 104.73 & 59.12 & 137.54 \\
\hline CD at $5 \%$ & 313.94 & 177.23 & 412.31 \\
\hline
\end{tabular}


Table.2 Effect of foliar application of compost tea on uptake of $\mathrm{N}$ in plant at different growth stage of soybean

\begin{tabular}{|c|c|c|c|c|c|c|c|c|c|}
\hline \multirow[t]{3}{*}{ Treatment } & \multirow{2}{*}{\multicolumn{2}{|c|}{ At flowering stage }} & \multirow{2}{*}{\multicolumn{2}{|c|}{$\begin{array}{l}\text { At pod formation } \\
\text { stage }\end{array}$}} & \multicolumn{4}{|c|}{ At harvesting stage } & \multirow[b]{3}{*}{$\begin{array}{c}\text { Total } \\
\text { uptake } \\
\left(\mathrm{kgha}^{-1}\right)\end{array}$} \\
\hline & & & & & \multicolumn{2}{|c|}{ Straw } & \multicolumn{2}{|c|}{ seed } & \\
\hline & $\begin{array}{c}\text { Content } \\
\mathrm{N} \\
(\%)\end{array}$ & $\begin{array}{c}\text { Uptake } \\
\mathrm{N} \\
\left(\mathrm{kg} \mathrm{ha}^{-1}\right)\end{array}$ & $\begin{array}{c}\text { Content } \\
\mathrm{N} \\
(\%)\end{array}$ & $\begin{array}{l}\text { Uptake } \\
\mathrm{N} \\
\left(\mathrm{kg} \mathrm{ha}^{-1}\right)\end{array}$ & $\begin{array}{c}\text { Content } \\
\mathrm{N} \\
(\%)\end{array}$ & $\begin{array}{c}\text { Uptake } \\
\mathrm{N} \\
\mathrm{Kg} \mathrm{ha}_{1}{ }^{-}\end{array}$ & $\begin{array}{c}\text { Content } \\
\mathrm{N} \\
(\%)\end{array}$ & $\left.\begin{array}{c}\text { Uptake } \\
\mathrm{N} \\
\left(\mathrm{kgha}^{-}\right. \\
1\end{array}\right)$ & \\
\hline $\mathrm{T}_{1}-$ Control (RDF 100\%) & 2.91 & 54.67 & 1.69 & 60.25 & 1.25 & 39.02 & 4.50 & 71.12 & 110.14 \\
\hline$T_{2}-100 \%$ RDF + Water spray & 2.94 & 55.74 & 1.70 & 61.43 & 1.26 & 39.99 & 4.55 & 71.77 & 111.75 \\
\hline$\underset{\text { spray }}{\mathrm{T}_{3}-\mathrm{RDF}+\text { Compost tea } @ 10 \% \text { foliar }}$ & 3.03 & 59.61 & 1.76 & 65.69 & 1.30 & 42.50 & 4.69 & 77.69 & 120.19 \\
\hline $\begin{array}{c}\text { T4 - RDF + Compost tea @ } 15 \% \text { foliar } \\
\text { spray }\end{array}$ & 3.07 & 62.95 & 1.78 & 69.37 & 1.32 & 44.80 & 4.75 & 82.32 & 127.12 \\
\hline 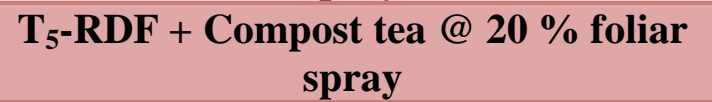 & 3.11 & 67.40 & 1.80 & 74.28 & 1.34 & 48.06 & 4.81 & 87.84 & 135.90 \\
\hline $\mathrm{T}_{6}-\mathrm{RDF}+\underset{\text { Compost tea } @ 25 \% \text { foliar }}{\text { spray }}$ & 3.17 & 72.07 & 1.84 & 79.42 & 1.36 & 51.27 & 4.91 & 94.35 & 145.62 \\
\hline $\mathbf{T}_{7}-\mathrm{RDF}+\underset{\text { spray }}{\text { Compost tea @ } 50 \% \text { foliar }}$ & 3.25 & 78.05 & 1.89 & 86.01 & 1.40 & 55.27 & 5.03 & 103.09 & 158.35 \\
\hline $\mathrm{T}_{8}-\mathrm{RDF}+\underset{\text { Compost tea } @ 75 \% \text { foliar }}{\text { spray }}$ & 3.22 & 75.28 & 1.86 & 82.96 & 1.38 & 53.03 & 4.98 & 100.44 & 153.47 \\
\hline General mean & 3.09 & 65.72 & 1.79 & 72.43 & 1.33 & 46.74 & 4.78 & 86.08 & 132.82 \\
\hline $\mathrm{SE}(\mathbf{m}) \pm$ & 0.068 & 2.971 & 0.04 & 3.274 & 0.029 & 2.212 & 0.106 & 3.823 & 5.89 \\
\hline CD at $5 \%$ & 0.204 & 8.906 & 0.119 & 9.814 & 0.088 & 6.632 & 0.316 & 11.461 & 17.657 \\
\hline
\end{tabular}


Table.3 Effect of foliar application of compost tea on uptake of $\mathrm{P}$ in plant at different growth stage of soybean

\begin{tabular}{|c|c|c|c|c|c|c|c|c|c|}
\hline \multirow[t]{3}{*}{ Treatment } & \multirow{2}{*}{\multicolumn{2}{|c|}{ At flowering stage }} & \multirow{2}{*}{\multicolumn{2}{|c|}{$\begin{array}{l}\text { At pod formation } \\
\text { stage }\end{array}$}} & \multicolumn{4}{|c|}{ At harvesting stage } & \multirow{3}{*}{$\begin{array}{c}\text { Total } \\
\text { uptake } \\
\left(\mathrm{kgha}^{-1}\right)\end{array}$} \\
\hline & & & & & \multicolumn{2}{|c|}{ Straw } & \multicolumn{2}{|c|}{ seed } & \\
\hline & $\begin{array}{c}\text { Content } \\
\mathrm{P} \\
(\%)\end{array}$ & $\begin{array}{c}\text { Uptake } \\
\mathrm{P} \\
\left(\mathrm{kg} \mathrm{ha}^{-1}\right)\end{array}$ & $\begin{array}{c}\text { Content } \\
\mathrm{P} \\
(\%)\end{array}$ & $\begin{array}{c}\text { Uptake } \\
\mathrm{P} \\
\left(\mathrm{kg} \mathrm{ha}^{-1}\right)\end{array}$ & $\begin{array}{l}\text { Content P } \\
\qquad \%)\end{array}$ & $\begin{array}{c}\text { Uptake } \\
\mathrm{P} \\
\left.\mathrm{Kg} \mathrm{ha}^{-1}\right)\end{array}$ & $\begin{array}{c}\text { Content } \\
\mathrm{P} \\
(\%)\end{array}$ & $\begin{array}{c}\text { Uptake } \\
\mathrm{P} \\
\left(\mathrm{kgha}^{-1}\right)\end{array}$ & \\
\hline T1 - Control (RDF 100\%) & 0.24 & 4.53 & 0.18 & 4.99 & 0.09 & 2.86 & 0.59 & 9.41 & 12.26 \\
\hline $\mathrm{T}_{2}-100 \%$ RDF + Water spray & 0.24 & 4.55 & 0.18 & 5.02 & 0.09 & 2.89 & 0.60 & 9.41 & 12.30 \\
\hline $\begin{array}{c}\mathrm{T}_{3}-\mathrm{RDF}+\text { Compost tea } @ 10 \\
\% \text { foliar spray }\end{array}$ & 0.25 & 4.92 & 0.19 & 5.42 & 0.1 & 3.1 & 0.65 & 10.69 & 13.79 \\
\hline $\begin{array}{c}\text { T4 - RDF + Compost tea @ } \\
\text { 15\% foliar spray }\end{array}$ & 0.27 & 5.54 & 0.20 & 6.10 & 0.11 & 3.85 & 0.65 & 11.52 & 15.37 \\
\hline $\begin{array}{c}\mathrm{T}_{5}-\mathrm{RDF}+\text { Compost tea @ } 20 \\
\% \text { foliar spray }\end{array}$ & 0.29 & 6.29 & 0.21 & 6.93 & 0.12 & 4.13 & 0.68 & 12.92 & 17.05 \\
\hline $\begin{array}{c}\mathrm{T}_{6}-\mathrm{RDF}+\text { Compost tea @ } 25 \\
\% \text { foliar spray }\end{array}$ & 0.31 & 7.04 & 0.23 & 7.76 & 0.13 & 5.06 & 0.73 & 14.4 & 19.46 \\
\hline $\begin{array}{c}\mathrm{T}_{7} \text { - RDF + Compost tea @ } \mathbf{5 0} \\
\% \text { foliar spray }\end{array}$ & 0.33 & 7.88 & 0.26 & 8.69 & 0.17 & 6.72 & 0.77 & 16.51 & 23.23 \\
\hline $\begin{array}{c}\text { T }_{8} \text { - RDF + Compost tea @ } 75 \\
\text { \% foliar spray }\end{array}$ & 0.32 & 7.51 & 0.24 & 8.27 & 0.15 & 5.62 & 0.75 & 15.51 & 21.14 \\
\hline General mean & 0.28 & 6.03 & 0.21 & 6.65 & 0.12 & 4.28 & 0.69 & 12.55 & 16.82 \\
\hline $\mathbf{S E}(\mathbf{m}) \pm$ & 0.009 & 0.257 & 0.006 & 0.283 & 0.005 & 0.244 & 0.021 & 0.604 & 0.773 \\
\hline CD at $5 \%$ & 0.028 & 0.769 & 0.017 & 0.849 & 0.015 & 0.73 & 0.062 & 1.809 & 2.318 \\
\hline
\end{tabular}


Table.4 Effect of foliar application of compost tea on uptake of $\mathrm{K}$ in plant at different growth stage of soybean

\begin{tabular}{|c|c|c|c|c|c|c|c|c|c|}
\hline \multirow[t]{3}{*}{ Treatment } & \multirow{2}{*}{\multicolumn{2}{|c|}{ At flowering stage }} & \multirow{2}{*}{\multicolumn{2}{|c|}{$\begin{array}{c}\text { At pod formation } \\
\text { stage }\end{array}$}} & \multicolumn{4}{|c|}{ At harvesting stage } & \multirow{3}{*}{$\begin{array}{c}\text { Total } \\
\text { uptake } \\
\left(\text { kgha }^{-1}\right)\end{array}$} \\
\hline & & & & & & & & & \\
\hline & $\begin{array}{c}\text { Content } \\
\mathrm{k} \\
(\%)\end{array}$ & $\begin{array}{c}\text { Uptake } \\
\mathrm{k} \\
\left(\mathrm{kg} \mathrm{ha}^{-1}\right)\end{array}$ & $\begin{array}{c}\text { Content } \\
\mathrm{k} \\
(\%)\end{array}$ & $\begin{array}{c}\text { Uptake } \\
\mathrm{K} \\
\left(\mathrm{kg} \mathrm{ha}^{-1}\right)\end{array}$ & $\begin{array}{c}\text { Content } \\
\mathrm{K} \\
(\%)\end{array}$ & $\begin{array}{c}\text { Uptake } \\
\mathrm{K} \\
\left.\mathrm{Kg} \mathrm{ha}^{-1}\right)\end{array}$ & $\begin{array}{c}\text { Content } \\
\mathrm{K} \\
(\%)\end{array}$ & $\begin{array}{c}\text { Uptake } \\
\mathrm{K} \\
\left(\mathrm{kgha}^{-1}\right)\end{array}$ & \\
\hline $\mathrm{T}_{1}$ - Control (RDF 100\%) & 1.29 & 24.22 & 1.01 & 35.9 & 0.75 & 23.32 & 1.41 & 22.19 & 45.50 \\
\hline$T_{2}-100 \%$ RDF + Water spray & 1.30 & 24.72 & 1.02 & 36.63 & 0.76 & 23.92 & 1.42 & 22.41 & 46.33 \\
\hline $\begin{array}{c}\mathrm{T}_{3}-\mathrm{RDF}+\text { Compost tea @ } 10 \% \\
\text { foliar spray }\end{array}$ & 1.38 & 27.06 & 1.07 & 40.1 & 0.80 & 26.02 & 1.50 & 24.83 & 50.85 \\
\hline $\begin{array}{c}\text { T4 - RDF + Compost tea @ 15\% } \\
\text { foliar spray }\end{array}$ & 1.42 & 29.13 & 1.11 & 43.17 & 0.83 & 27.96 & 1.55 & 26.82 & 54.78 \\
\hline $\begin{array}{c}\mathrm{T}_{5}-\mathrm{RDF}+\text { + Compost tea @ } 20 \% \\
\text { foliar spray }\end{array}$ & 1.47 & 31.9 & 1.15 & 47.28 & 0.85 & 30.66 & 1.61 & 29.32 & 59.98 \\
\hline $\begin{array}{c}\mathrm{T}_{6}-\mathrm{RDF}+\text { Compost tea @ } 25 \% \\
\text { foliar spray }\end{array}$ & 1.51 & 34.23 & 1.18 & 50.73 & 0.87 & 32.87 & 1.64 & 31.5 & 64.37 \\
\hline $\begin{array}{c}\mathbf{T}_{7}-\mathrm{RDF}+\text { Compost tea @ } 50 \% \\
\text { foliar spray }\end{array}$ & 1.57 & 37.71 & 1.22 & 55.89 & 0.91 & 35.99 & 1.71 & 35.14 & 71.13 \\
\hline $\begin{array}{c}\mathrm{T}_{8}-\mathrm{RDF}+\text { Compost tea } @ 75 \\
\text { \% foliar spray }\end{array}$ & 1.53 & 35.74 & 1.19 & 52.97 & 0.89 & 33.96 & 1.66 & 33.58 & 67.53 \\
\hline General mean & 1.43 & 30.59 & 1.12 & 45.33 & 0.83 & 29.34 & 1.56 & 28.22 & 57.56 \\
\hline $\mathrm{SE}(\mathrm{m}) \pm$ & 0.032 & 1.101 & 0.025 & 1.629 & 0.018 & 1.164 & 0.035 & 1.234 & 2.078 \\
\hline CD at $5 \%$ & 0.095 & 3.299 & 0.074 & 4.882 & 0.055 & 3.491 & 0.104 & 3.699 & 6.228 \\
\hline
\end{tabular}


Table.5 Balance sheet of available nitrogen $\left(\mathrm{N} \mathrm{kg} \mathrm{ha}^{-1}\right)$ in soil as influenced foliar application of compost tea on soybean

\begin{tabular}{|c|c|c|c|c|c|c|c|}
\hline Sr. No & $\begin{array}{c}\text { Initial } \\
\text { fertilizer status } \\
\left(\mathrm{kg} \mathrm{ha}^{-1}\right)\end{array}$ & $\begin{array}{c}\text { Added } \\
\text { Nitrogen } \\
\left(\mathrm{kg} \mathrm{ha}^{-1}\right)\end{array}$ & $\begin{array}{c}\text { Nitrogen } \\
\text { uptake } \\
\left(\mathrm{kg} \mathrm{ha}^{-1}\right)\end{array}$ & $\begin{array}{c}\text { Expected } \\
\text { balance } \\
\left(\mathrm{kg} \mathrm{ha}^{-1}\right)\end{array}$ & $\begin{array}{c}\text { Actual } \\
\text { fertility status } \\
\left(\mathrm{kg} \mathrm{ha}^{-1}\right)\end{array}$ & $\begin{array}{c}\text { Apparent } \\
\text { gain/loss } \\
\left(\mathbf{k g ~ h a}^{-1}\right)\end{array}$ & $\begin{array}{c}\text { Actual } \\
\text { gain/loss } \\
\left(\mathbf{k g ~ h a}^{-1}\right)\end{array}$ \\
\hline & (A) & (B) & (C) & $(D)=(A+B)-(C)$ & (E) & $(F)=(E-D)$ & $(\mathbf{G})=(\mathbf{E}-\mathbf{A})$ \\
\hline $\mathbf{T}_{1}$ & 210.35 & 30 & 110.14 & 130.21 & 249.87 & 119.66 & 39.52 \\
\hline $\mathbf{T}_{2}$ & 210.35 & 30 & 111.75 & 128.60 & 248.19 & 119.59 & 37.84 \\
\hline $\mathbf{T}_{3}$ & 210.35 & 30 & 120.19 & 120.16 & 241.09 & 120.93 & 30.74 \\
\hline $\mathbf{T}_{4}$ & 210.35 & 30 & 127.12 & 113.23 & 236.4 & 123.17 & 26.05 \\
\hline $\mathbf{T}_{5}$ & 210.35 & 30 & 135.90 & 104.45 & 227.31 & 122.86 & 16.96 \\
\hline $\mathbf{T}_{6}$ & 210.35 & 30 & 145.62 & 94.73 & 215.54 & 120.81 & 5.19 \\
\hline $\mathbf{T}_{7}$ & 210.35 & 30 & 158.35 & 82.00 & 203.57 & 121.57 & -6.78 \\
\hline $\mathbf{T}_{8}$ & 210.35 & 30 & 153.47 & 86.88 & 209.48 & 122.60 & -0.87 \\
\hline
\end{tabular}

Table.6 Balance sheet of available phosphorus $\left(\mathrm{P} \mathrm{kg} \mathrm{ha}^{-1}\right)$ in soil as influenced foliar application of compost tea on soybean

\begin{tabular}{|c|c|c|c|c|c|c|c|}
\hline Sr.No & $\begin{array}{c}\text { Initial } \\
\text { fertilizer status } \\
\left(\mathrm{kg} \mathrm{ha}^{-1}\right)\end{array}$ & $\begin{array}{c}\text { Added } \\
\text { phosphorus } \\
\left(\mathrm{kg} \mathrm{ha}^{-1}\right)\end{array}$ & $\begin{array}{c}\text { phosphorus } \\
\text { uptake } \\
\left(\mathbf{k g ~ h a}^{-1}\right)\end{array}$ & $\begin{array}{l}\text { Expected } \\
\text { balance } \\
\left(\mathrm{kg} \mathrm{ha}^{-1}\right)\end{array}$ & $\begin{array}{c}\text { Actual } \\
\text { fertility status } \\
\left(\mathrm{kg} \mathrm{ha}^{-1}\right)\end{array}$ & $\begin{array}{c}\text { Apparent } \\
\text { gain/loss } \\
\left(\mathrm{kg} \mathrm{ha}^{-1}\right)\end{array}$ & $\begin{array}{c}\text { Actual } \\
\text { gain/loss } \\
\left(\mathrm{kg} \mathrm{ha}^{-1}\right)\end{array}$ \\
\hline & (A) & (B) & (C) & $(D)=(A+B)-(C)$ & (E) & $(F)=(E-D)$ & $(G)=(E-A)$ \\
\hline $\mathbf{T}_{1}$ & 19.54 & 60 & 12.26 & 67.28 & 23.42 & -43.86 & 3.88 \\
\hline $\mathbf{T}_{2}$ & 19.54 & 60 & 12.30 & 67.24 & 23.19 & -44.05 & 3.65 \\
\hline $\mathbf{T}_{3}$ & 19.54 & 60 & 13.79 & 65.75 & 22.59 & -43.16 & 3.05 \\
\hline $\mathbf{T}_{4}$ & 19.54 & 60 & 15.37 & 64.17 & 22.40 & -41.77 & 2.86 \\
\hline $\mathbf{T}_{5}$ & 19.54 & 60 & 17.05 & 62.49 & 21.28 & -41.20 & 1.74 \\
\hline $\mathbf{T}_{6}$ & 19.54 & 60 & 19.46 & 60.08 & 19.91 & -40.17 & 0.37 \\
\hline $\mathbf{T}_{7}$ & 19.54 & 60 & 23.23 & 56.31 & 18.20 & -38.11 & -1.34 \\
\hline $\mathbf{T}_{8}$ & 19.54 & 60 & 21.14 & 58.40 & 18.72 & -39.68 & -0.82 \\
\hline
\end{tabular}


Table.7 Balance sheet of available Potassium $\left(\mathrm{kg} \mathrm{ha}^{-1}\right)$ in soil as influenced foliar application of compost tea on soybean

\begin{tabular}{|c|c|c|c|c|c|c|c|}
\hline Sr.No & $\begin{array}{c}\text { Initial } \\
\text { fertilizer } \\
\text { status } \\
\left(\mathbf{k g ~ h a}^{-1}\right)\end{array}$ & $\begin{array}{c}\text { Added } \\
\text { Potassium } \\
\left(\mathrm{kg} \mathrm{ha}^{-1}\right)\end{array}$ & $\begin{array}{c}\text { Potassium } \\
\text { uptake } \\
\left(\mathbf{k g ~ h a}^{-1}\right)\end{array}$ & $\begin{array}{c}\text { Expected } \\
\text { balance } \\
\left(\mathrm{kg} \mathrm{ha}^{-1}\right)\end{array}$ & $\begin{array}{c}\text { Actual } \\
\text { fertility } \\
\text { status } \\
\left(\mathbf{k g ~ h a}^{-1}\right)\end{array}$ & $\begin{array}{c}\text { Apparent } \\
\text { gain/loss } \\
\left(\mathbf{k g ~ h a}^{-1}\right)\end{array}$ & $\begin{array}{c}\text { Actual } \\
\text { gain/loss } \\
\left(\mathrm{kg} \mathrm{ha}^{-1}\right)\end{array}$ \\
\hline & (A) & (B) & (C) & $\begin{array}{c}(D)=(A+B)- \\
(C)\end{array}$ & (E) & $(F)=(E-D)$ & $\begin{array}{c}(\mathbf{G})=(\mathbf{E}- \\
\text { A) }\end{array}$ \\
\hline $\mathbf{T}_{1}$ & 467.73 & 30 & 45.50 & 452.23 & 506.28 & 54.05 & 38.55 \\
\hline $\mathbf{T}_{2}$ & 467.73 & 30 & 46.33 & 451.40 & 503.60 & 52.19 & 35.87 \\
\hline $\mathbf{T}_{\mathbf{3}}$ & 467.73 & 30 & 50.85 & 446.88 & 502.40 & 55.53 & 34.67 \\
\hline $\mathbf{T}_{4}$ & 467.73 & 30 & 54.78 & 442.95 & 493.99 & 51.04 & 26.26 \\
\hline $\mathbf{T}_{5}$ & 467.73 & 30 & 59.98 & 437.75 & 488.77 & 51.02 & 21.04 \\
\hline$T_{6}$ & 467.73 & 30 & 64.37 & 433.36 & 484.58 & 51.22 & 16.85 \\
\hline $\mathbf{T}_{7}$ & 467.73 & 30 & 71.13 & 426.60 & 475.81 & 49.20 & 8.08 \\
\hline $\mathbf{T}_{8}$ & 467.73 & 30 & 67.53 & 430.20 & 478.52 & 48.33 & 10.79 \\
\hline
\end{tabular}

Apparent balance of phosphorus $\left(\mathrm{P}_{2} \mathrm{O}_{5}\right)$ status of soil $\left(\mathrm{kg} \mathrm{ha}^{-1}\right)$

Data on available phosphorus $\left(\mathrm{P}_{2} \mathrm{O}_{5}\right)$ status after harvest of soybean as influenced by foliar application of compost tea when studied revealed that the irrespective of treatment combinations a definite gain or loss in available phosphorus status of soil after harvest of soybean crop was observed. Maximum gain in available phosphorus status $\left(3.88 \mathrm{~kg} \mathrm{ha}^{-1}\right)$ was registered in the treatment $\mathrm{T}_{1}$-control receiving $(100 \% \mathrm{RDF}$ through chemical fertilizers while more loss of available $\mathrm{P}\left(-1.34 \mathrm{~kg} \mathrm{ha}^{-1}\right)$ was observed in the treatment $\left(\mathrm{T}_{7}\right)$ where $\mathrm{RDF}+\mathrm{T}_{7}-\mathrm{RDF}+$ Compost tea @ $50 \%$ foliar spray. In general increasing the dose of foliar applied compost tea on soybean A decrease in available nitrogen status of soil after harvest of the soybean crop.

\section{Apparent balance potassium $\left(\mathrm{K}_{2} \mathrm{O}\right)$ status of soil $\left(\mathrm{kg} \mathrm{ha}^{-1}\right)$}

Data on available potassium $\left(\mathrm{K}_{2} 0\right)$ status after harvest of brinjal as influenced by organic manures and inorganic fertilizers when studied revealed that the irrespective of treatment combinations a definite gain in available potassium status of soil after harvest of soybean crop was observed.

Maximum gain in available potassium status $\left(38.55 \mathrm{~kg} \mathrm{ha}^{-1}\right)$ was registered in the treatment $\mathrm{T}_{1}$-control receiving (100\% $\mathrm{RDF}$ through chemical fertilizers while minimum $(8.08 \mathrm{~kg}$ $\left.\mathrm{ha}^{-1}\right)$ was observed in the treatment $\left(\mathrm{T}_{7}\right)$ where $\mathrm{RDF}+\mathrm{T}_{7}-\mathrm{RDF}+$ Compost tea @ $50 \%$ foliar spray. In general increasing the dose of foliar applied compost tea on soybean A decrease in available potassium status of soil after harvest of the soybean crop.

Maximum yield (2050.0 kg ha ${ }^{-1}$ ) was recorded with application of RDF + Compost tea @ 50 $\%$ foliar spray. Significantly higher Uptake of N,P,K and DTPA extractable micro nutrient $(\mathrm{Cu}, \mathrm{Fe}, \mathrm{Zn}, \mathrm{Mn})$ was found in soybean applied with RDF + Compost tea @ 50\% foliar spray over the control.

Significantly higher Uptake of N,P,K was found in soybean applied with RDF + Compost tea @ 50\% foliar spray. Foliar application of 30:60:30 kg ha- $\mathrm{N}: \mathrm{P}_{2} \mathrm{O}_{5}: \mathrm{K}_{2} \mathrm{O}$ with foliar application of compost tea @ 50 
percent $(30,45,60 \mathrm{DAS})$ to soybean was found beneficial for increase in balance sheet of available nutrients.

\section{References}

Das, P. C., 1999, Text book of manures and fertilizers, kalyani publisher. pp 46-47.

Frankenberger, W. T. and Arshad, M(1995). Phytormones in soil. Marcel dekker Inc.New York.,335-71.

Hegazi, A.Z. and Algharib, A.M. (2014) Studied the utilizing compost tea as a nutrient amendment in open field cowpea seed production system. J. Bio and Env. Sci. 2, 318-328.

Hiradeve, N. P., Deotale, R. D., Deogirkar, S. M. and Gaikwad, B. S. (2014) Effectivity of foliar sprays of vermicompost wash on chemical, biochemical, yield and yield contributing parameters of groundnut. $J$. Soil and Crops 21(2), 266-272.

Ingham, E. R (2000) Compost tea Brewing Manual. Soil Food Web Incorporated. Corvallis, Oregon, USA, pp 59-63

Ingham, E. R (2005) Compost tea Brewing Manual. Latest Methods and Research Soil Food Web.

Jackson, M. L. (1967) Soil Chemical Analysis, Prentice Hall of India Pvt. Ltd., New Delhi

Kumawat, R. N., Mahajan, S. S. and Mertia, R. S. (2009) Responce of cumin to panchgavya and leaf extracts in arid western Rajasthan. J. spice and Aromatic Crops.18 (2): 9299.
Maheswari, V. N., Kaleena, P. K., Srikumaran, M. P, Rekha, G. S., and Elumalai, D. (2003) Influence of vermiwash and panchagavya on lablab beans under pot experimental conditions. Int. J. Adv. Res. Biol. Sci. 4(2): 20-27.

Mostafa, M. F. M., El-Boray, M. S. S., Abd Elwahab, A. F. and Barakat, R.A. (2014) Effect of enriched compost tea on Washington navel orange trees. J. Agric. Sci. Mansoura Univ. 34, 10085-10094.

Piper, C. S. (1966) Soil and Plant Analysis. Hans publishers, Bombay, p. 368.

Rathore, S. S., Chaudhary, D. R., Boricha, G. N., Ghosh, A., Bhatt, B.P., Zodape, S. T. and Patolia J. S. (2009) Studied the effects of foliar applications of different concentrations of seaweed extract prepared from (Kappaphycus alvarezii) on nutrient uptake, growth and yield of soybean grown under rainfed conditions. South African J. Botany. 75, 351-355.

Scheuerell, S. J. and Mahaffee, W. F. (2002). Compost tea principals and prospects for plant disease control. Compost Sci. and Utilization 10, 313-338.

Scheuerell, S. J. and Mahaffee, W. F. (2004) Compost tea as container medium drench for suppressing seedling damping-off caused by (pythium ultimatum). Phytopathology. 94, 1156-1163.

Zodape, S. T., Kawarkhe, V. J., Patolia, J. S. and Warade, A. D. (2008) Effect of liquid seaweed fertilizer on yield and quality of okra (Abelmoschus esculentus). J. Scientific \& Industrial Res. 69, 1115-1117.

\section{How to cite this article:}

Shrinivas, N., P. H. Vaidya and Gourkhede, P. H. 2021. Effect of Foliar Application of Compost Tea on Soil Quality, Nutrient Uptake and Apparent Nutrient Balance in Soil under Soybean [Glycine max (L)]. Int.J.Curr.Microbiol.App.Sci. 10(05): 594-604. doi: https://doi.org/10.20546/ijcmas.2021.1005.067 